\title{
ARQUITECTURA Y MORFOMETRÍA DE DOS ESPECIES ARBÓREAS EN una selva baja caducifolia del sur de Yucatán, MÉxico
}

\author{
Víctor Manuel Interián-Ku¹, 5 , Juan Ignacio Valdez-Hernández², Edmundo García-Moya", \\ Angélica Romero-Manzanares ${ }^{1}$, María Amparo Borja-de-la-Rosa ${ }^{3}$ y Humberto Vaquera-Huerta ${ }^{4}$ \\ ${ }^{1}$ Colegio de Postgraduados, Programa en Botánica. \\ ${ }^{2}$ Colegio de Postgraduados, Programa Forestal. Carretera México-Texcoco km 36.5. \\ Código Postal 56230. Montecillo, Estado de México. \\ ${ }^{3}$ Universidad Autónoma Chapingo. División de Ciencias Forestales. Km. 38.5 carretera México-Texcoco. \\ Chapingo, Estado de México. \\ ${ }^{4}$ Colegio de Postgraduados, Programa en Estadística. \\ ${ }^{5}$ Autor para la correspondencia. Correo-e: interian@colpos.mx
}

\begin{abstract}
Resumen: En una selva baja caducifolia de Yucatán, México, se describió la arquitectura y se analizó los atributos dasométrricos de Caesalpinia gaumeri y Gymnopodium floribundum en tres clases de tamaño y dos tipos de suelo. Caesalpinia gaumeri se utiliza en construcción rural y $G$. floribundum en apicultura. Se identificaron y clasificaron 24 individuos de ambas especies en tres clases de tamaño: brinzales, latizales y fustales y en suelos luvisol y leptosol. La arquitectura de C. gaumeri correspondió al modelo Troll, mientras que G. floribundum al Champagnat. En ambas especies, se observaron reiteraciones relacionadas a causas naturales. Se encontró diferencias estadísticas para los atributos dasométricos entre clases de tamaños y entre tipos de suelo para ambas especies. Los árboles fustales de $C$. gaumeri tuvieron mayores (Tukey, $P \leq 0.05$ ) diámetros a la altura del pecho, alturas totales de copa y a la primera rama, longitudes de rama de primer orden y de entrenudos entre clases de tamaño. Los individuos de esta especie que crecen en suelo luvisol cuentan con mayores (Tukey, $P \leq 0.05$ ) valores de diámetro a la altura del pecho y ángulo de inserción de ramas de tercer orden a diferencia de los ubicados en leptosol. Los árboles brinzales de G. floribundum tuvieron ángulos de inserción de ramas de primer orden mayores (Tukey, $P \leq 0.05$ ) al de los latizales y fustales. Los individuos de esta especie que crecen en suelo luvisol tuvieron mayores (Tukey, $P \leq 0.05$ ) ángulos de inserción de ramas de segundo y tercer orden en comparación con los que crecen en leptosol.
\end{abstract}

Palabras clave: Caesalpinia gaumeri, Gymnopodium floribundum, clases de tamaño, mediciones dasométricas, tipos de suelo, análisis discriminante canónico.

\begin{abstract}
In a low deciduous forest in Yucatan, Mexico, we describe the architecture and analyzer the morphometry attributes of Caesalpinia gaumeri and Gymnopodium floribundum in three size classes and two soil types. Caesalpinia gaumeri is used in rural construction and G. floribundum in beekeeping. We identified and classified 24 trees of both species in three size classes: seedlings, saplings and poles, in soil luvisol and leptosol. The architecture of C. gaumeri corresponded to the model Troll and G. floribundum is Champagnat. Both species showed reiterations related of natural causes. We found statistics differences for morphometry attributes between size classes and soil types for both species. The tree poles of $C$. gaumeri were higher (Tukey, $P \leq 0.05)$ in diameter at breast height, total height of crown and total height a first branch, lengths of branch of first order and internodes between size classes. The trees of this species growth in soil luvisol were higher (Tukey, $P \leq 0.05$ ) in diameter at breast height and insertion angle of branches of third order compared with trees growth in leptosol. The trees seedlings of $G$. floribundum were higher (Tukey, $P \leq 0.05$ ) in insertion angle of branches of first order compared with saplings and poles. The trees of this species growth in soil luvisol were higher (Tukey, $P \leq 0.05$ ) in insertion angle of branches of second and third order compared with trees growth in leptosol.
\end{abstract}

Keywords: Caesalpinia gaumeri, Gymnopodium floribundum, size-classes, forestry measurements, soil types, canonical discriminant analysis.

$\mathbf{L}^{2}$ a arquitectura vegetal se estudia a través de dibujos sobre un plano, los cuales permiten llegar a modelar la morfología de las plantas (Tomlinson, 1983), bajo la premisa de que la expresión morfológica de estas es resultante de la interacción entre el genotipo y el medio que le rodea por lo que es posible encontrar una gran variación morfológica entre especies e identificar uno o más patrones de crecimiento (Hallé et al., 1978; Barthélémy y Caraglio, 2007). 
En especies forestales, conocer la morfología puede ser una herramienta útil para el diseño de plantaciones (Altieri $e t$ al., 1983) y el mejor aprovechamiento de bosques naturales mediante la aplicación de técnicas silvícolas (Meza y Torres, 2004; Muñoz et al., 2008).

Los trabajos pioneros en este campo corresponden a Hallé y Oldeman (1970), Oldeman (1974) y Hallé et al. (1978); los realizados en el trópico mexicano pertenecen a Torquebiau (1981) con especies de Veracruz, a Echevery-Gómez (2000) y Vester (2002) con algunas especies arbóreas de Yucatán y a Baltazar-Ramírez (2001) y Echevery-Gómez y Vester (2001) con especies de Quintana Roo. Son escasos los trabajos sobre indicadores de los cambios en los patrones morfológicos entre clases de tamaño, como el de Jiménez-Rojas et al. (2002) quienes estudiaron los procesos de transición y reiteración entre éstas y que, inclusive incorporaron análisis estadísticos para caracterizar efectos de supresión y predominio en árboles del Amazonas. En especies económicamente importantes, se ha encontrado que la arquitectura de los árboles se relaciona con la eficiencia y la producción de madera, como por ejemplo en el tallo de Pinus contorta var. Latifolia (Smith y Long, 1989) y con los patrones de floración y fructificación por ejemplo en manzano y mango (Lauri et al., 2006; Normand et al., 2009).

El presente trabajo se centra en la expresión morfológica de dos especies arbóreas en una selva baja caducifolia del sur de Yucatán: Caesalpinia gaumeri Greenm (en maya kitimché) y Gymnopodium floribundum Rolfe (en maya tzitzilché) para las cuales, White y Hood (2004) encontraron altos valores de densidad (372 y 400 árboles ha ${ }^{-1}$, respectivamente) y área basal (13.4 y $7.7 \mathrm{~m} 2 \mathrm{ha}^{-1}$, respectivamente) en Ticul, Yucatán, a $7 \mathrm{~km}$ del área de estudio, así como contrastes en la composición estructural de la vegetación arbórea que se desarrolla en suelos pedregosos de Calketok, Yucatán y no pedregosos de Othón P. Blanco, Quintana Roo.

Particularmente, Caesalpinia gaumeri, por las características mecánicas y de resistencia a la pudrición de su madera, se utiliza para la construcción doméstica de casas estilo maya (25\% del total de la madera usada en una casa habitación), así como para leña (100 a $120 \mathrm{~kg}$ por semana por familia) y carbón (15.8\% del total empleado en un horno tradicional) (Levy-Tacher y Hernández-Xolocotzi, 1994). Es un árbol que pertenece a la familia Fabaceae, subfamilia Caesalpinoideae y puede alcanzar hasta $13 \mathrm{~m}$ de altura, con profundas acanaladuras y perforaciones en el tronco, la corteza forma placas pequeñas y rectilíneas; tiene hojas alternas imparipinnadas con foliolo liso; la inflorescencia es un racimo con flores amarillas de simetría bilateral; el pétalo central se encuentra cubierto con manchas naranjas; el fruto es una legumbre plana (Standley y Steyermark, 1946; Souza-Novelo, 1981; Sosa et al., 1985).

Gymnopodium floribundum es la especie más importante del estado de Yucatán en la producción comercial de miel
(Echazarreta et al., 1997; Ramírez-Cervantes et al., 2000; Moguel-Ordóñez et al., 2005) y en el año 2003 contribuyó, junto con otras especies (por ejemplo: Viguiera dentata (Cav.) Spreng.), con $14.7 \%$ al total de la producción nacional (INEGI, 2005). Es un árbol que pertenece a la familia Polygonaceae, con tres metros de alto o más, con corteza profundamente fisurada, hojas alternas y simples, de ovadas a elípticas, peciolo corto, base redondeada a obtusa, margen completo, ápice redondo a obtuso, con pubescencia en el envés; las flores son panículas racemosas de color verdoso y se desarrollan de yemas que aparecen al final o al principio de la estación de crecimiento (Standley y Steyermark, 1946; Souza-Novelo, 1981; Sosa et al., 1985).

Dada la importancia de Caesalpinia gaumeri y Gymnopodium floribundum, y con el propósito de contribuir al conocimiento de la morfología en ambas especies, el objetivo del presente trabajo fue describir su arquitectura y analizar su morfometría en tres diferentes clases de tamaño $\mathrm{y}$ dos tipos de suelo.

Las hipótesis planteadas son las siguientes: a) se esperan encontrar diferencias entre clases de tamaño y entre tipos de suelo para los atributos dasométricos medidos en las especies estudiadas, y b) no existen modificaciones de la morfología en las especies estudiadas debidas a causas naturales.

\section{Materiales y métodos}

La presente investigación se llevó a cabo en el predio particular "San Manuel" ubicado a $90 \mathrm{~km}$ al sur de Mérida, a $20^{\circ} 23^{\prime} 46^{\prime \prime}$ de latitud Norte, $89^{\circ} 26^{\prime} 19$ ' de longitud Oeste y $24 \mathrm{msnm}$, en el municipio de Dzan, Yucatán, localizado en una selva baja caducifolia de aproximadamente 60 años sin disturbio humano y con árboles de más de $11 \mathrm{~m}$ de altura. En forma aleatoria, se establecieron seis unidades de muestreo (UM) de $10 \times 20 \mathrm{~m}$ (White y Hood, 2004) en dos condiciones edáficas: tres UM en k'ankab (luvisol) y otras tres en tzek'el (leptosol). Los leptosoles de la parte sur de Yucatán, son generalmente pobres en nutrimentos aunque ricos en materia orgánica ( 36 a 45\%) con alta concentración de carbonatos de calcio (4 a 12\%). Los luvisoles no cuentan con rocas $(0 \%)$, tienen buena profundidad $(80 \mathrm{~cm})$ y retención de humedad y poca materia orgánica (4 a 12\%) en comparación con los leptosoles, y no presentan carbonatos de calcio (García et al., 2005).

En las UM, se identificaron y marcaron todos los individuos de Caesalpinia gaumeri y Gymnopodium floribundum libres de plagas o enfermedades y se seleccionaron ocho individuos por clase de tamaño con base en la clasificación modificada de Valdez (2002): brinzales ( $<1.5 \mathrm{~m}$ de altura), latizales $[\geq 1.5 \mathrm{~m}$ de altura y hasta $2.5 \mathrm{~cm}$ de diámetro a la altura del pecho (DAP) medido a $1.3 \mathrm{~m}$ sobre el suelo] y fustales ( $>2.5 \mathrm{~cm}$ de DAP), con un total de 24 árboles por especie. Para conocer el modelo arquitectónico que mejor se ajusta a las especies en estudio, se consideraron las siguien- 
tes variables morfológicas por individuo: Crecimiento del eje principal: Patrón -monopódico o simpódico, Forma -determinado o indeterminado, Actividad -proléptico o siléptico; Ramificación: Patrón -monopodial, simpodial monocasio o simpodial dicasio, Orientación y simetría -ortotrópico, plagiotrópico o combinado, Ordenamiento -eje principal orden 1 y en sucesión orden 2, orden 3 , etc., Ángulo de inserción de ramas -ascendente $\left(16\right.$ a $\left.45^{\circ}\right)$ o difuso $\left(46\right.$ a $\left.75^{\circ}\right)$, Longitud de ramas -largas o cortas, Longitud de entrenudos -largos o macroblastos, cortos o braquiblastos; Estructuras reproductivas: Posición -axilar o apical, Tipo -monopodiales complejas como panículas o simpodiales cimoides clase monocasio, dicasio o pleiocasio; Reiteraciones: total o parcial, adaptativa o traumática, proléptica o siléptica. Para complementar esta información se realizaron dibujos del individuo por clase de tamaño y también, de ramas y hojas.

La terminología morfológica ya ha sido definida por autoridades en la materia (Tomlinson y Gill, 1973; McMahon, 1975; Tomlinson, 1978, 1987; Hallé et al., 1978; Brayton, 1984; Moreno, 1984; Osada y Takeda, 2003; Barthélémy y Caraglio, 2007) pero para facilitar el acceso a esta información, se presenta en un glosario (anexo A).

Las mediciones dasométricas incluyeron: diámetro del fuste a $1.30 \mathrm{~m}$ de altura, altura total, altura a la primera rama, altura de copa, longitud de ramas de primer, segundo y tercer orden, longitud de entrenudos, longitud de lámina y base de la hoja y ángulo de inserción de ramas de primer, segundo y tercer orden. Con los resultados se realizaron análisis de varianza (ANOVA), comparaciones de medias y un análisis de discriminante canónico (ADC), mediante el paquete estadístico SAS (SAS Institute Inc., 1999).

\section{Resultados}

Caesalpinia gaumeri. Se caracteriza por diferenciarse en tronco y rama, el eje principal tiene un crecimiento simpódico e indeterminado con ejes plagiotrópicos que se superponen de manera continua (Figura 1). El patrón de ramificación es simpodial, plagiotrópico y siléptico, sin embargo, en individuos brinzales y latizales las ramas de la parte inferior del tallo, aparecen y después de cierto tiempo se secan y caen conforme el eje principal crece en altura (RS y RC en Figuras 1 b y 1c), hasta conformar un fuste sin ramas por debajo del dosel, justo antes de pasar a la etapa denominada fustal (Figura 1a). La orientación de la rama de primer orden en relación al eje principal es ascendente para los brinzales y latizales $\left(31.2\right.$ y $\left.36.6^{\circ}\right)$ y difusa para los fustales $\left(51^{\circ}\right)$ (Cuadro 1). Las inflorescencias se presentan en racimos con

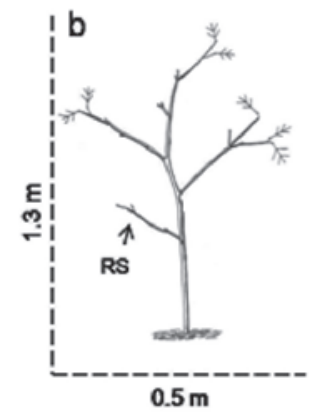

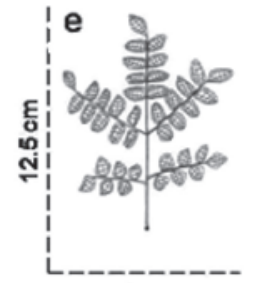

$11 \mathrm{~cm}$



Figura 1. Arquitectura de Caesalpinia gaumeri: a) fustal, polieje diferenciado en tronco y ramas con reiteración traumática (R); b) brinzal, ejes plagiotrópicos y ramas secas (RS); c) latizal, eje principal simpodial con ramificación monocasio; ramas bajas se secarán y caerán (RC); d) rama con órdenes de ramificación sucesivos (1,2,3) plagiotrópicos, crecimiento simpodial monocasio y siléptico; e) hoja dística imparipinnada. 
VÍCTOR MANUEL INTERIÁN-Ku, ET AL.

Cuadro 1. Cuadrados medios del análisis de varianza para atributos dasométricos de Caesalpinia gaumeri.

\begin{tabular}{|c|c|c|c|c|c|c|c|c|}
\hline Fuente de variación & gl & DAP & AT & ALCOP & APRA & ARPO & ARSO & ARTO \\
\hline Clases de tamaño & 2 & $428.71^{* * *}$ & $235.24^{* * *}$ & $54.10^{* * *}$ & $66.56^{* * *}$ & $841.34^{*}$ & $539.13 \mathrm{~ns}$ & $215.57 \mathrm{~ns}$ \\
\hline Suelo & 1 & $9.54 *$ & $0.4333 \mathrm{~ns}$ & $1.25 \mathrm{~ns}$ & $0.1320 \mathrm{~ns}$ & $17.59 \mathrm{~ns}$ & $536.85 \mathrm{~ns}$ & $1506.38^{*}$ \\
\hline BFL(SUE) & 2 & $8.74^{*}$ & $0.5404 \mathrm{~ns}$ & $0.1958 \mathrm{~ns}$ & $0.1094 \mathrm{~ns}$ & 204.18ns & $123.73 \mathrm{~ns}$ & $257.09 \mathrm{~ns}$ \\
\hline Error & 18 & 1.75 & 0.4554 & 0.5204 & 0.6313 & 232.63 & 589.11 & 311.79 \\
\hline Fuente de variación & gl & LRPO & LRSO & LRTO & \multicolumn{2}{|c|}{ LENT } & LLHO & LBHO \\
\hline Clases de tamaño & 2 & $317824.02^{* * *}$ & $1840.33 n s$ & \multicolumn{2}{|l|}{$2559.50^{* *}$} & $17923.57^{* * *}$ & $13.89 \mathrm{~ns}$ & $4.16 n s$ \\
\hline Suelo & 1 & $1160.65 \mathrm{~ns}$ & $2353.03 \mathrm{~ns}$ & \multicolumn{2}{|l|}{$852.76 \mathrm{~ns}$} & $2262.04 \mathrm{~ns}$ & $1.91 \mathrm{~ns}$ & $0.0135 \mathrm{~ns}$ \\
\hline $\mathrm{BFL}(\mathrm{SUE})$ & 2 & $2193.49 n s$ & $847.24 \mathrm{~ns}$ & \multicolumn{2}{|l|}{$219.39 n s$} & $764.69 n s$ & $1.94 \mathrm{~ns}$ & $2.56 \mathrm{~ns}$ \\
\hline Error & 18 & 2082.79 & 894.22 & \multicolumn{2}{|l|}{377.68} & 1661.51 & 5.81 & 3.74 \\
\hline
\end{tabular}

$\mathrm{gl}=$ grados de libertad; $\mathrm{BFL}(\mathrm{SUE})=$ clases de tamaño dentro de tipos de suelo; DAP = diámetro en $\mathrm{cm}$, a $1.3 \mathrm{~m}$ de altura; $\mathrm{AT}=$ altura total del árbol en $\mathrm{m} ; \mathrm{ALCOP}=$ altura de copa en $\mathrm{m} ; \mathrm{APRA}=$ altura a primera rama en m; $\mathrm{ARPO}$ = ángulo de inserción de rama de primer orden en grados; ARSO = ángulo de inserción de rama de segundo orden en grados; ARTO = ángulo de inserción de rama de tercer orden en grados; LRPO = longitud de rama de primer orden en cm; LRSO = longitud de rama de segundo orden en cm; LRTO = longitud de rama de tercer orden en $\mathrm{cm}$; LENT = longitud de entrenudos en cm; LLHO = longitud de la lámina de la hoja en cm; LBHO = longitud de la base de la hoja en $\mathrm{cm}$. Diferencias altamente significativas $\left(* * *=P \leq 0.001 \mathrm{y}^{* *}=P \leq 0.01\right)$; Diferencias significativas $(*=P \leq 0.05) ; \mathrm{ns}=\sin$ diferencias significativas $(P>0.05)$.

posición axilar, de desarrollo acrópeto y filotaxia helicoidal (Figura 2a); las flores son amarillas zigomorfas con manchas de color naranja en el pétalo central y hermafroditas; las primeras defloran y caen y sólo las apicales se transforman en fruto (Figura 2b). Las hojas jóvenes (Figura 2a) presentan una coloración rojiza por antocianinas que no se observa en las maduras (Figura 2b).

Es común encontrar lesiones y cavidades en la parte interna del tronco en la clase fustal como respuesta al daño causado por el alojamiento de larvas y hormigas adultas ho'och (Pachycondyla villosa Fabricius) y modificaciones morfológicas a manera de reiteraciones traumáticas silépticas
(R en Figura 1a) como respuesta al daño físico de las ramas.

El análisis de varianza entre clases de tamaño mostró diferencias altamente significativas $(P \leq 0.001)$ en los atributos dasométricos: DAP, altura total, altura de copa y a la primera rama, longitud de rama de primer orden y longitud de entrenudo, y significativas $(P \leq 0.05)$ para el ángulo de inserción de rama de primer orden y longitud de rama de tercer orden (Cuadro 1). Se encontraron diferencias significativas $(P \leq 0.05)$ para DAP y ángulo de inserción de rama de tercer orden entre tipos de suelo y sólo para DAP entre clases de tamaño dentro de tipo de suelo (Cuadro 1).

Respecto a los atributos dasométricos por clases de tama-



Figura 2. Caesalpinia gaumeri: a) racimos b) frutos. 
ARQUITECTURA Y MORFOMETRÍA DE ÁRBOLES TROPICALES.

Cuadro 2. Valores promedio de atributos dasométricos para Caesalpinia gaumeri.

\begin{tabular}{|c|c|c|c|c|c|c|c|c|}
\hline Clases de tamaño & $\mathbf{N}$ & DAP & AT & ALCOP & APRA & ARPO & ARSO & ARTO \\
\hline Brinzal & 8 & $0.671 b$ & $0.994 \mathrm{c}$ & $0.597 \mathrm{C}$ & $0.361 b$ & $* 31.21 \mathrm{~b}$ & $65.22 \mathrm{a}$ & $58.26 \mathrm{a}$ \\
\hline Latizal & 8 & $1.32 \mathrm{~b}$ & $2.87 \mathrm{~b}$ & $1.65 b$ & $1.00 \mathrm{~b}$ & *36.56ba & $49.76 a$ & $52.80 \mathrm{a}$ \\
\hline Fustal & 8 & $13.66 \mathrm{a}$ & $11.18 \mathrm{a}$ & $5.53 a$ & $5.65 a$ & $* * 51.03 a$ & $62.27 a$ & $47.88 \mathrm{a}$ \\
\hline DMSH & & 1.69 & 0.8611 & 0.9206 & 1.0139 & 19.46 & 30.97 & 22.53 \\
\hline \multicolumn{9}{|l|}{ Tipo de suelo } \\
\hline K'ankab & 12 & $5.85 a$ & $5.15 \mathrm{a}$ & $2.82 \mathrm{a}$ & $2.26 a$ & $40.46 a$ & $54.35 a$ & $60.91 a$ \\
\hline Tzek'el & 12 & $4.59 \mathrm{~b}$ & $4.88 \mathrm{a}$ & $2.37 \mathrm{a}$ & $2.41 \mathrm{a}$ & $38.74 a$ & $63.81 \mathrm{a}$ & $45.06 \mathrm{~b}$ \\
\hline DMS & & 1.13 & 0.579 & 0.619 & 0.682 & 13.08 & 20.81 & 15.14 \\
\hline Clases de tamaño & $\mathbf{N}$ & LRPO & LRSO & LRTO & & LENT & LLHO & LBHO \\
\hline Brinzal & 8 & $26.30 b$ & $36.39 a$ & $18.16 b$ & & $17.31 b$ & $13.04 a$ & $9.32 \mathrm{a}$ \\
\hline Latizal & 8 & $62.31 b$ & $49.92 a$ & $25.04 b$ & & $22.19 b$ & $15.31 \mathrm{a}$ & $10.10 \mathrm{a}$ \\
\hline Fustal & 8 & $388.13 a$ & $66.66 a$ & $52.00 \mathrm{a}$ & & 101.63a & $13.01 \mathrm{a}$ & $8.66 a$ \\
\hline DMSH & & 58.24 & 38.16 & 24.80 & & 52.01 & 3.07 & 2.47 \\
\hline \multicolumn{9}{|l|}{ Tipo de suelo } \\
\hline K'ankab & 12 & $165.87 a$ & $41.09 a$ & $25.77 a$ & & $56.75 a$ & $13.50 \mathrm{a}$ & $9.34 \mathrm{a}$ \\
\hline Tzek'el & 12 & $151.96 a$ & $60.89 b$ & $37.70 \mathrm{a}$ & & $37.33 a$ & $14.07 a$ & $9.38 \mathrm{a}$ \\
\hline DMS & & 39.14 & 25.65 & 16.67 & & 34.96 & 2.07 & 1.66 \\
\hline
\end{tabular}

$\mathrm{N}$ = número de individuos en cada clase. DMS = diferencia mínima significativa.

Valores seguidos por letras iguales entre hileras no son estadísticamente diferentes (Tukey, $P>0.05$ ).

Orientación con respecto al eje principal: ascendente $\left(^{*}\right)$ y difuso $\left(^{* *}\right)$. Ver significado de variables en cuadro 1.

ño, los fustales presentaron los mayores valores promedio (Tukey, $P \leq 0.05$ ) de DAP, altura total de copa y a la primera rama, longitudes de rama de primer y tercer orden y de entrenudos, mientras que los brinzales y latizales tuvieron promedios menores de altura total y de copa así como ángulo de inserción de rama de primer orden (Cuadro 2). Respecto a los tipos de suelo, los individuos que crecen en luvisol cuentan con mayores valores promedio de DAP y ángulo de inserción de rama de tercer orden y los que crecen en leptosol tienen mayores longitudes de rama de segundo orden (Cuadro 2).

$\mathrm{El}$ análisis discriminante canónico (ADC) para los atributos dasométricos indicó que las primeras dos funciones discriminantes (FD) explicaron el 99\% de la variación total (Figura 3). Las variables que contribuyeron a determinar la FD1 $(96.5 \%)$ fueron: altura total, longitud de rama de primer orden, DAP, altura a la primera rama y altura de copa, lo cual indica que el plano arquitectónico del árbol se imprime desde el principio de las etapas de desarrollo, privilegiando la expresión de esos caracteres para alcanzar el estrato superior. Para la FD2 (2.5\%) los atributos de importancia fueron: ángulo de inserción de rama de tercer orden y longitud de rama de segundo orden, cualidades relacionadas con la anchura de copa. La figura 3 muestra una clara separación entre fustales y latizales, pero no así entre latizales y brinzales. Sin embargo, los latizales que crecen en suelos luvisol se separan de los brinzales. De acuerdo con las significancias

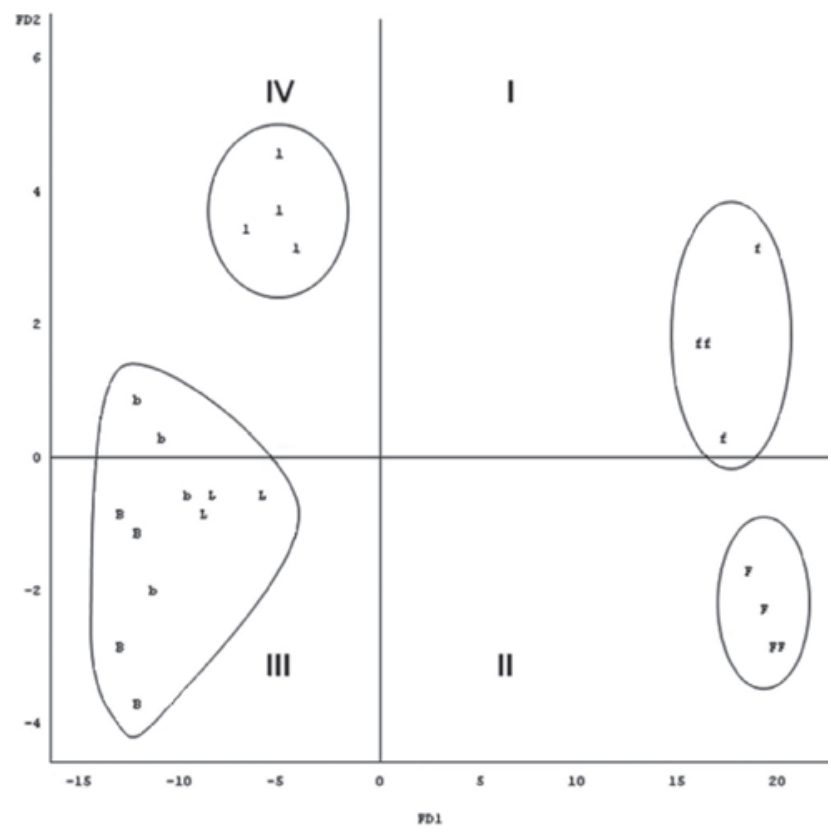

Figura 3. Distribución de Caesalpinia gaumeri (24 individuos) con base en las primeras dos funciones discriminantes (FD1, FD2): brinzales en suelos k'ankab (B) y tzek'el (b), latizales en suelos k'ankab (L) y tzek'el (1), fustales en suelos k'ankab (F) y tzek'el (f). 
Víctor MANuel InTERiÁN-Ku, ET AL.

Cuadro 3. Valores de probabilidad de la distancia de Mahalanobis entre clases de tamaño dentro de tipos de suelo para Caesalpinia gaumeri.

\begin{tabular}{|c|c|c|c|c|c|c|c|}
\hline \multirow[t]{2}{*}{ Clases de tamaño } & & \multicolumn{2}{|l|}{ Brinzal } & \multicolumn{2}{|l|}{ Latizal } & \multicolumn{2}{|l|}{ Fustal } \\
\hline & & $K^{\prime} a n k a b$ & Tzek'el & K'ankab & Tzek'el & K'ankab & Tzek'el \\
\hline \multirow[t]{2}{*}{ Brinzal } & K'ankab & ----- & & & & & \\
\hline & Tzek'el & $45.42 \mathrm{~ns}$ & ----- & & & & \\
\hline \multirow[t]{2}{*}{ Latizal } & K'ankab & $1.55 \mathrm{~ns}$ & $0.8659 \mathrm{~ns}$ & ----- & & & \\
\hline & Tzek'el & $4.68^{*}$ & $2.80 \mathrm{~ns}$ & $1.35 \mathrm{~ns}$ & ----- & & \\
\hline \multirow[t]{2}{*}{ Fustal } & K'ankab & $52.02 * * *$ & $47.12^{* * *}$ & $37.75^{* * *}$ & $32.48^{* * *}$ & ----- & \\
\hline & Tzek'el & $45.48^{* * *}$ & $40.29^{* * *}$ & $32.06^{* * *}$ & $25.73^{* * *}$ & $1.49 \mathrm{~ns}$ & ----- \\
\hline
\end{tabular}

*** diferencias altamente significativas $(P \leq 0.001), *$ diferencias significativas $(P \leq 0.05), \mathrm{ns}=\sin$ diferencias significativas $(P>0.05)$.

estadísticas del cuadro 3, no existe una separación de individuos por tipos de suelo dentro de clase de tamaño.

Gymnopodium floribundum. Presenta un patrón de crecimiento simpódico, indeterminado y siléptico del eje principal, por superposición indefinida de ejes dorsiventrales mixtos (plagiotrópicos y ortotrópicos), con filotaxia helicoidal (Figura 4). Cada eje distal de relevo cuelga por su propio peso (Figuras 4c y 4d), el renuevo se origina en la parte superior al inicio de la curva del eje colgante (Figura 4c). Los retoños en la parte distal del eje se convierten en ramas mientras que los proximales forman parte del tronco. El patrón de ramificación es simpodial monocasio, de orientación combinada y siléptico, la orientación de la rama de primer orden con respecto al eje principal es ascendente para latizal y fustal $\left(36.6\right.$ y $51^{\circ}$ ) y difusa para brinzal $\left(31.2^{\circ}\right.$ ) (Cuadro $2)$. Es común encontrar reiteraciones traumáticas parenquimatosas en forma de nudos bayoneta (RNB) en ramas de primer orden (Figuras 4b, 4c y 4d), como respuesta a los
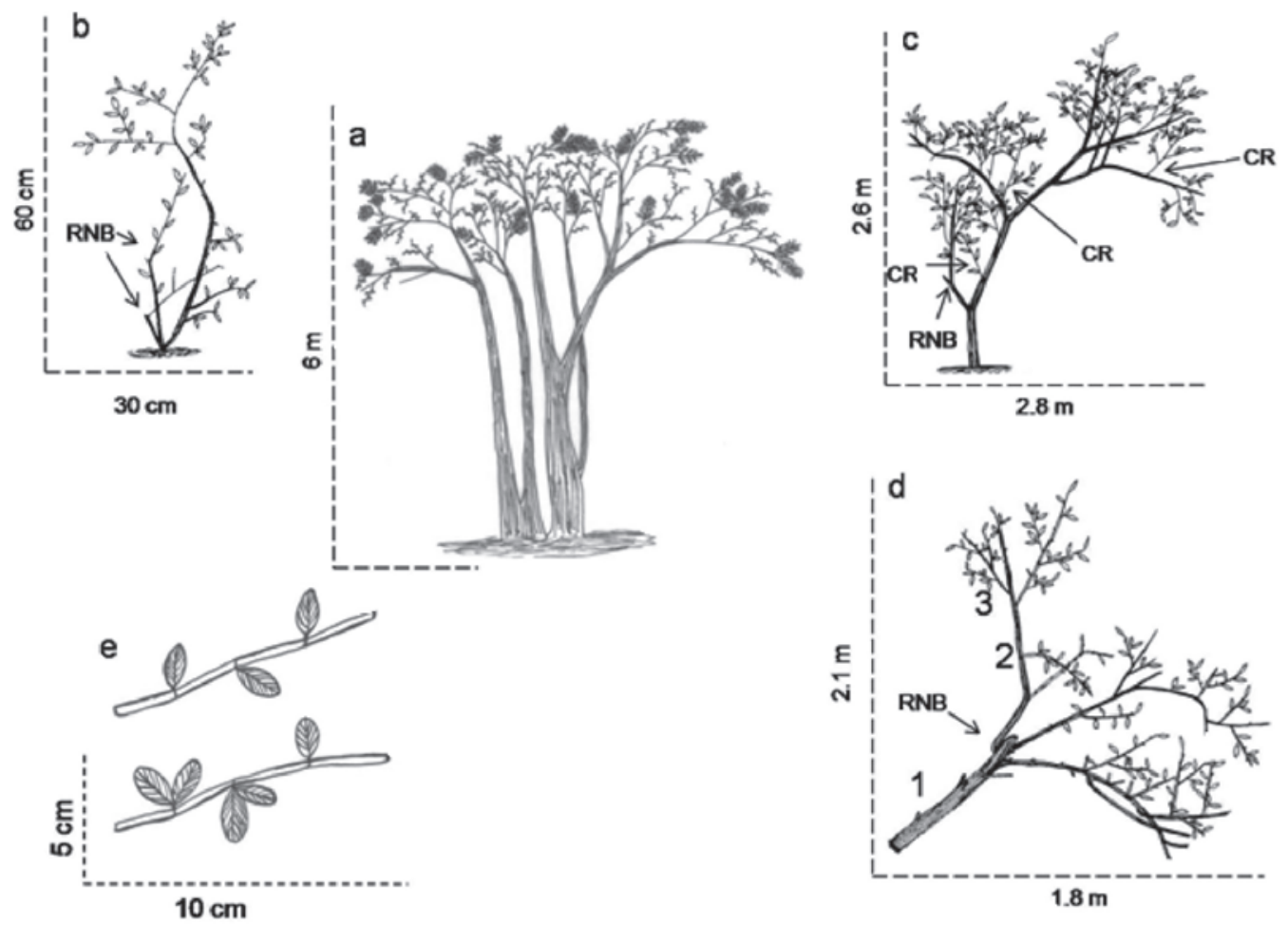

Figura 4. Arquitectura de Gymnopodium floribundum: a) fustal poliejes; b) brinzal, de crecimiento simpódico monocasio alternado, con reiteraciones traumáticas que muestran nudo bayoneta (RNB); c) latizal, con desarrollo simpodial dicasio y la parte distal colgante mientras que la proximal es sustituyente del tronco o eje principal con crecimiento simpodial monocasio y reiteraciones traumáticas con nudo bayoneta (RNB) más complejos de reiteraciones (CR); d) rama con dicasios, primer, segundo y tercer orden $(1,2,3)$; e) filotaxia alterna y una a dos hojas por nudo. 
múltiples daños físicos a los que está expuesta esta especie por encontrarse en el estrato medio-bajo del dosel y complejos de reiteraciones (CR) adaptativas en ramas de primer y segundo orden como parte del proceso natural de crecimiento de los latizales (Figura 4c). Presenta inflorescencias tipo panículas racemosas que se desarrollan en forma acrópeta y se estructuran en filotaxia helicoidal; las flores son trímeras y hermafroditas (Figura 5).

El análisis de varianza indica que existen diferencias altamente significativas $(P \leq 0.01, P \leq 0.001)$ y significativas $(P \leq 0.05)$ entre clases de tamaño, entre tipos de suelo, así como de clases de tamaño dentro de tipo de suelo (Cuadro 4).

El cuadro 5 muestra que los brinzales tuvieron copas más abiertas en comparación con fustales y latizales porque sus ángulos de inserción de ramas de segundo orden fueron significativamente mayores (Tukey, $P \leq 0.05$ ). Los individuos establecidos en suelos luvisol tuvieron ramas de segundo y tercer orden con mayores ángulos de inserción (Tukey, $P \leq$ $0.05)$ y los establecidos en suelos leptosol tuvieron mayores longitudes de rama de segundo y tercer orden.

El ADC indicó que las primeras dos funciones discriminantes (FD) explicaron el 97.6\% de la variación total (Figura 6). Las variables que contribuyeron a determinar la FD1 $(94.4 \%)$ fueron: DAP, altura total, altura de copa y longitud de entrenudos; mientras que para la FD2 (3.2\%), la longitud y el ángulo de inserción de rama de segundo orden fueron las de mayor importancia. La figura 6 muestra una clara separación entre fustales, latizales y brinzales y para fustales entre tipos de suelo. Lo anterior está apoyado por los resultados que se muestran en el cuadro 6.

Ambas especies no mostraron metamorfosis, es decir, cambios en el modelo arquitectónico inducidos por cambios en la etapa de desarrollo.

\section{Discusión}

De acuerdo con los patrones de crecimiento y ramificación de estas dos especies en la etapa adulta, Caesalpinia gau-



Figura 5. Inflorescencias tipo panículo-racemosa de Gymnopodium floribundum

meri adopta el modelo Troll como todas las leguminosas, el cual se construye mediante la sobreposición continua de ejes plagiotrópicos. Los ejes principales próximos que crecen erectos construyen parte del tronco y los ejes distales con o sin crecimiento determinado forman parte de una rama (Hallé et al., 1978). Gymnopodium floribundum se expresa morfológicamente conforme al modelo Champagnat el cual se caracteriza por la superposición de ejes mixtos ortótropos. Los próximos forman un tronco simpódico y los distales se vuelven péndulos y forman las ramas. (Hallé $e t$ al., 1978). En ambos casos esta clasificación coincide con lo reportado por Vester (2002).

La caída de ramas que crecen sobre el eje principal en brinzales y latizales de Caesalpinia gaumeri, producto de autopodas, reduce la amplitud de la copa y forma un tallo limpio en los fustales (Mäkelä y Valentine, 2006), característica que funcionalmente favorece a plantas de estratos inferiores, porque permite difundir la luz solar y aprovechar los gradientes de radiación (Küppers, 1989), mientras que en términos de aprovechamiento, la autopoda puede reducir

Cuadro 4. Cuadrados medios del análisis de varianza para atributos dasométricos de Gymnopodium floribundum.

\begin{tabular}{|c|c|c|c|c|c|c|c|c|}
\hline Fuente de variación & gl & DAP & AT & ALCOP & APRA & ARPO & ARSO & ARTO \\
\hline Clases de tamaño & 2 & $189.09^{* * *}$ & $80.06^{* * *}$ & $36.83^{* * *}$ & $8.26^{* * *}$ & $993.64 n s$ & $1042.07^{* *}$ & $247.89 n s$ \\
\hline Suelo & 1 & $29.88^{* * *}$ & $0.683 \mathrm{~ns}$ & $3.56^{* *}$ & $1.13 \mathrm{~ns}$ & $1032.02 \mathrm{~ns}$ & $1226.51^{* *}$ & $3186.89^{* *}$ \\
\hline BFL(SUE) & 2 & $33.26 * * *$ & $4.28^{* *}$ & $3.34^{* *}$ & $0.8701 \mathrm{~ns}$ & $328.17 \mathrm{~ns}$ & $529.42^{*}$ & $433.92 \mathrm{~ns}$ \\
\hline Error & 18 & 0.918 & 0.520 & 0.417 & 0.401 & 368.24 & 145.04 & 257.88 \\
\hline Fuente de variación & gl & LRPO & LRSO & LRTO & \multicolumn{2}{|c|}{ LENT } & LLHO & LBHO \\
\hline Clases de tamaño & 2 & $12368.81^{* *}$ & $7564.56^{* *}$ & $995.78 \mathrm{~ns}$ & \multicolumn{2}{|c|}{$273695.43^{* * *}$} & $10.52^{*}$ & $0.0765^{*}$ \\
\hline Suelo & 1 & $4449.93 \mathrm{~ns}$ & $12299.86^{* *}$ & $2159.35^{*}$ & \multicolumn{2}{|c|}{ 7568.60ns } & $0.9243 \mathrm{~ns}$ & $0.0737 \mathrm{~ns}$ \\
\hline BFL(SUE) & 2 & $4957.93 *$ & $1060.64 \mathrm{~ns}$ & 140.80ns & \multicolumn{2}{|c|}{ 8196.10ns } & $0.1195 \mathrm{~ns}$ & $0.0313 \mathrm{~ns}$ \\
\hline Error & 18 & 1184.87 & 855.33 & 416.78 & \multicolumn{2}{|c|}{4797.11} & 1.76 & 0.0184 \\
\hline
\end{tabular}

$\mathrm{gl}$ = grados de libertad; BFL(SUE) = clases de tamaño dentro de tipo de suelo. Diferencias altamente significativas $\left({ }^{* * *}=P \leq 0.001 \mathrm{y} * *=P \leq 0.01\right)$; Diferencias significativas $(*=P \leq 0.05)$; ns $=$ sin diferencias significativas $(P>0.05)$. Ver significado de variables en cuadro 1 . 
Víctor Manuel Interián-Ku, ET AL.

Cuadro 5. Valores promedio de atributos dasométricos para Gymnopodium floribundum.

\begin{tabular}{|c|c|c|c|c|c|c|c|c|}
\hline Clases de tamaño & $\mathbf{N}$ & DAP & AT & ALCOP & APRA & ARPO & ARSO & ARTO \\
\hline Brinzal & 8 & $0.610 \mathrm{~b}$ & $1.03 c$ & $0.699 \mathrm{c}$ & $0.339 b$ & $* 53.66 a$ & $70.36 a$ & $64.16 a$ \\
\hline Latizal & 8 & $1.80 \mathrm{~b}$ & $3.34 b$ & $2.29 b$ & $1.05 b$ & $* * 34.00 \mathrm{a}$ & $48.75 b$ & $53.24 \mathrm{a}$ \\
\hline Fustal & 8 & $9.56 a$ & $7.29 a$ & $4.94 a$ & $2.34 \mathrm{a}$ & $* * 34.74 a$ & $53.20 \mathrm{~b}$ & $60.58 a$ \\
\hline DMS & & 1.22 & 0.920 & 0.824 & 0.808 & 24.49 & 15.37 & 20.49 \\
\hline \multicolumn{9}{|l|}{ Tipo de suelo } \\
\hline K'ankab & 12 & $5.11 b$ & $3.72 \mathrm{a}$ & $2.26 b$ & $1.46 \mathrm{a}$ & $47.36 a$ & $64.59 \mathrm{a}$ & $70.85 a$ \\
\hline Tzek'el & 12 & $2.87 \mathrm{a}$ & $4.06 a$ & $3.03 a$ & $1.03 a$ & $34.25 a$ & $50.29 \mathrm{~b}$ & $47.81 \mathrm{~b}$ \\
\hline DMS & & 0.822 & 0.618 & 0.554 & 0.543 & 16.46 & 10.33 & 13.77 \\
\hline
\end{tabular}

Continuación cuadro 5 ...

\begin{tabular}{|c|c|c|c|c|c|c|c|}
\hline Clases de tamaño & $\mathbf{N}$ & LRPO & LRSO & LRTO & LENT & LLHO & LBHO \\
\hline Brinzal & 8 & $10.70 \mathrm{a}$ & $23.94 b$ & $19.07 \mathrm{a}$ & $35.40 \mathrm{~b}$ & $2.86 b$ & $0.320 \mathrm{~b}$ \\
\hline Latizal & 8 & $53.25 \mathrm{ba}$ & $81.54 a$ & $40.58 a$ & $87.50 b$ & $5.12 \mathrm{a}$ & $0.506 a$ \\
\hline Fustal & 8 & $89.25 b$ & $71.41 \mathrm{a}$ & $24.66 a$ & $378.63 a$ & $4.31 \mathrm{ba}$ & $0.465 \mathrm{ba}$ \\
\hline DMS & & 43.92 & 37.32 & 26.05 & 88.38 & 1.69 & 0.173 \\
\hline \multicolumn{8}{|l|}{ Tipo de suelo } \\
\hline K'ankab & 12 & $37.45 a$ & $36.32 b$ & $18.62 b$ & $149.42 \mathrm{a}$ & $4.29 a$ & $0.486 a$ \\
\hline Tzek'el & 12 & $64.68 a$ & $81.60 a$ & $37.59 a$ & $184.93 a$ & $3.89 a$ & $0.375 a$ \\
\hline DMS & & 29.52 & 25.08 & 17.51 & 59.40 & 1.14 & 0.116 \\
\hline
\end{tabular}

$\mathrm{N}$ = número de individuos en cada clase. DMS = diferencia mínima significativa.

Valores seguidos por letras iguales entre hileras no son estadísticamente diferentes (Tukey, $P>0.05$ ).

Orientación con respecto al eje principal: ascendente $\left.{ }^{*}\right)$ y difuso $\left.{ }^{* *}\right)$. Ver significado de variables en cuadro 1.

los costos de manejo silvícola. Las lesiones presentes en los troncos de los fustales ocasionadas por la hormiga Pachycondyla villosa y los daños físicos por la caída de ramas o árboles vecinos, generan cavidades en la parte interna del tallo y cicatrices en la cara externa, además de fustes poco cilíndricos, torcidos y deformes que afectan la calidad y valor económico de la madera potencialmente útil para las construcciones rurales, impide la fabricación de muebles y generan una corta selectiva hacia los individuos mejor conformados.
El análisis de varianza, la comparación de medias y el discriminante canónico sugieren que los individuos clasificados como brinzales y latizales en Caesalpinia gaumeri tienen copas más angostas debido a que la orientación de las ramas de primer orden con respecto al eje principal es ascendente, mientras que en fustales las ramas son más abiertas; lo anterior posibilita el crecimiento rápido en altura para alcanzar el dosel superior y luego expandir la copa para, en cierta forma, disipar el exceso calórico absorbido al recibir la radiación solar directa. Harold y Hocker (1984) indican

Cuadro 6. Valores de probabilidad de la distancia de Mahalanobis entre clases de tamaño dentro de tipos de suelo para Gymnopodium floribundum.

\begin{tabular}{|c|c|c|c|c|c|c|c|}
\hline \multirow[t]{2}{*}{ Clases de tamaño } & & \multicolumn{2}{|l|}{ Brinzal } & \multicolumn{2}{|l|}{ Latizal } & \multicolumn{2}{|l|}{ Fustal } \\
\hline & & K'ankab & Tzek'el & K'ankab & Tzek'el & K'ankab & Tzek'el \\
\hline Brinzal & $\begin{array}{l}\text { K'ankab } \\
\text { Tzek'el }\end{array}$ & $0.7186 \mathrm{~ns}$ & ------ & & & & \\
\hline Latizal & $\begin{array}{l}\text { K'ankab } \\
\text { Tzek'el }\end{array}$ & $\begin{array}{l}4.62^{*} \\
3.85 \mathrm{~ns}\end{array}$ & $\begin{array}{l}3.12 \mathrm{~ns} \\
2.65 \mathrm{~ns}\end{array}$ & $1.20 \mathrm{~ns}$ & ----- & & \\
\hline Fustal & $\begin{array}{l}\text { K'ankab } \\
\text { Tzek'el }\end{array}$ & $\begin{array}{l}26.35^{* * *} \\
77.59^{* * *}\end{array}$ & $\begin{array}{l}21.82 \mathrm{~ns} \\
69.70^{* * *}\end{array}$ & $\begin{array}{l}10.73^{* *} \\
46.90^{* * *}\end{array}$ & $\begin{array}{l}15.40^{* *} \\
54.34^{* * *}\end{array}$ & 17.44** & \\
\hline
\end{tabular}

Diferencias altamente significativas: ${ }^{* *} P \leq 0.001,{ }^{*} P \leq 0.01$; diferencias significativas: $* P \leq 0.05$; ns $=$ sin diferencias significativas. 


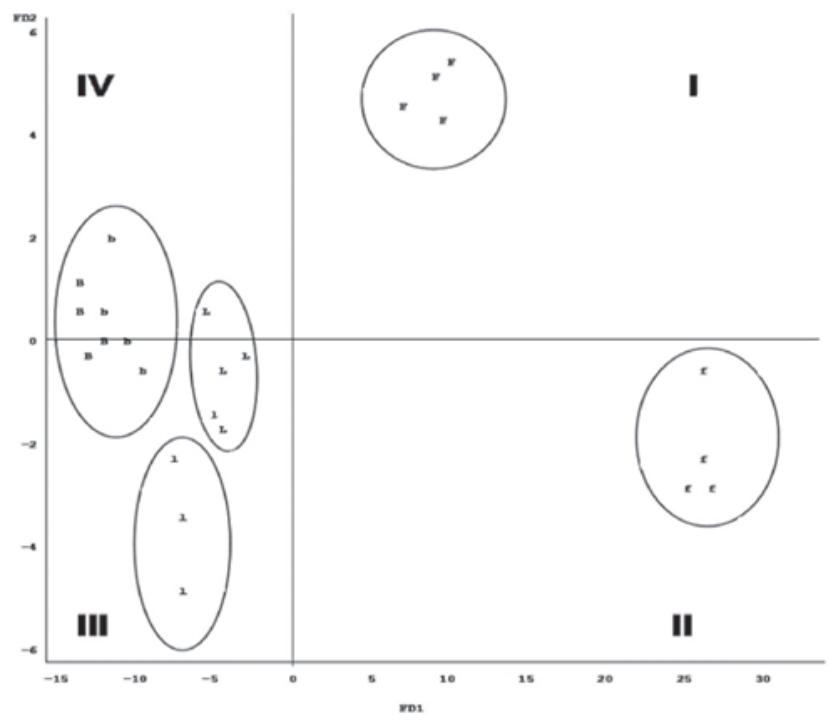

Figura 6. Distribución de Gymnopodium floribundum (24 individuos) con base en las primeras dos funciones discriminantes (FD1, FD2): brinzales en suelos k'ankab (B) y tzek'el (b), latizales en suelos k'ankab (L) y tzek'el (1), fustales en suelos k'ankab (F) y tzek'el (f).

que los árboles con necesidad de mayor cantidad y calidad de luz solar tratan de alcanzar el dosel superior lo que limita el crecimiento radial y la anchura de la copa.

En el caso de Gymnopodium floribundum como especie característica del estrato bajo a medio, los brinzales tienen copas más abiertas con ángulos de rama difusos, lo que les permite aprovechar mejor la escasa cantidad de luz que reciben en el sotobosque (Hallé et al., 1978, Poorter et al., 2006), mientras que los latizales y fustales que se encuentran en el estrato medio tienden a cerrar su copa por que las ramas de primer orden son ascendentes, lo que responde a la competencia por espacio, que es mayor entre adultos que entre jóvenes.

La comparación de medias indica, que los individuos de las dos especies que crecen en suelos luvisol tienen diámetros mayores pero copas compactas, a diferencia de los que crecen en suelos leptosol. Ledig (1983), explica que las plantas son capaces de adecuar su arquitectura conforme a las condiciones ambientales que le rodean para sobrevivir, y de acuerdo con los resultados observados en el presente trabajo las condiciones edáficas pueden afectarla, ya que como señalan Bruning y Sander (1983), a un suelo pobre en nutrimentos, compacto, salino y bajo sequía corresponden individuos de vástagos poco desarrollados y con una mayor expansión de la masa radical. Particularmente en el estado de Yucatán, White y Hood (2004), refieren que los suelos con menor porcentaje de rocas e inclinación de la pendiente (luvisol) favorecen el incremento del DAP en los individuos y la densidad de árboles por unidad de superficie, lo que genera competencia y obliga a reducir la amplitud de los doseles.

La posición que ocupan estas especies en el plano vertical de la estructura arbórea es complementaria, ya que Caesalpinia gaumeri se ubica en el estrato superior mientras que Gymnopodium floribundum en el medio-inferior, además de que presentan modelos arquitectónicos diferentes en cuanto a la apertura de copa; ambas características son importantes en términos prácticos, para el diseño de sistemas agroforestales.

Es evidente que los resultados del análisis morfológico indican similitudes arquitectónicas entre las tres etapas de desarrollo estudiadas dentro de especie, y diferencias contrastantes entre especies, lo que significa que el modelo arquitectónico está ontogénicamente determinado para cada especie, se mantiene constante entre etapas de desarrollo (brinzal, latizal y fustal), sólo se afecta dasométricamente por factores externos como el tipo de suelo, la cantidad de luz o la competencia interespecífica y que, como cualidad de respuesta al disturbio natural (alojamiento de hormigas) $o$ inducido (aprovechamiento de fustes y ramas), existe la tendencia a la reiteración estructural del organismo afectado.

\section{Conclusiones}

Las características morfológicas y patrones de ramificación observados, ratifican el ajuste de Caesalpinia gaumeri al modelo Troll y Gymnopodium floribundum al modelo Champagnat.

Se encontraron diferencias para los atributos dasométricos entre las clases de tamaño propuestas para cada especie y entre tipos de suelo, principalmente por el diámetro del fuste, alturas totales, de copa y a la primera rama; ángulos de inserción de ramas de segundo y tercer orden, longitudes de ramas de primer y segundo orden, longitud de entrenudos; todos, caracteres morfológicos que implican crecimiento en altura y amplitud de copa.

Contrario a lo planteado en la hipótesis "b", se encontraron diferencias en la morfología de las especies estudiadas a manera de reiteraciones, debidas a causas naturales tales como la caída de ramas de árboles vecinos y cavidades internas en el fuste de Caesalpinia gaumeri por el alojamiento de larvas y hormigas.

Por las características arquitectónicas, morfológicas y la ubicación en el estrato arbóreo vertical Caesalpinia gaumeri y Gymnopodium floribundum se podrían utilizar, de manera combinada, para el diseño de sistemas agroforestales para la obtención de madera y miel.

\section{Agradecimientos}

Al Consejo Nacional de Ciencia y Tecnología (CONACYT) por la beca otorgada (169577) para realizar estudios de doctorado y al Consejo Mexiquense de Ciencia y Tecnología 
(COMECYT) por la beca tesis (07BTD397) para concluir este documento.

\section{Literatura citada}

Altieri M.A., Letourneau D.K. y Davis. J.R. 1983. Developing sustainable agroecosystems. BioScience 33:45-49.

Baltazar-Ramírez J.O. 2001. Modelos arquitectónicos arbóreos en selva mediana subperennifolia secundaria y primaria, ejido X'Hazil Sur y anexos, Quintana Roo. Tesis de Maestría. El Colegio de la Frontera Sur. Chetumal, Quintana Roo. 27p.

Barthélémy D. y Caraglio Y. 2007. Plant architecture: a dynamic, multilevel and comprehensive approach to plant form, structure and ontogeny. Annals of Botany 99:375-407.

Bruning E.F. y Sander N. 1983. Ecosystem structure and functioning: some interactions of relevance to agroforestry. En: Huxley P.A Ed. Plant Research and Agroforestry. Nairobi, Kenya, pp. 221-247 International Council for Research in Agroforestry. Kenya.

Echevery-Gómez A. 2000. Desarrollo arquitectónico y variación morfológica de cuatro especies de leguminosas de la Península de Yucatán, México. Tesis de Maestría. El Colegio de la Frontera Sur. Chetumal, Quintana Roo. 46 p.

Echevery-Gómez A. y Vester H.F.M. 2001. Desarrollo arquitectónico de tres especies de Acacia. Boletín de la Sociedad Botánica de México 69:7-14.

García J., Mizrahi A. y Butista F. 2005. Manejo campesino de la selva baja y selección de especies arbóreas para barbechos mejorados en Hocabá, Yucatán. En: Bautista F y Palacio G Eds. Caracterización y manejo de los suelos de la Península de Yucatán: Implicaciones Agropecuarias, Forestales y Ambientales, pp. 195-208. Universidad Autónoma de Campeche, Universidad Autónoma de Yucatán.

Hallé F. y Oldeman R.A.A. 1970. Essai sur l'architecture et la dynamique de croissance des arbres tropicaux. Monographies de Botanique et de Biologie Végétale No. 6:1-178, Masson and Cie, Paris.

Hallé F., Oldeman R.A.A. y Tomlison P.B. 1978. Tropical trees and forests; an architectural analysis. Springer-Verlag. Berlin Heidelberg New York.

Harold y Hocker H.W. 1984. Introducción a la biología forestal. AGT Editor, S.A. México.

INEGI [Instituto Nacional de Estadística y Geografía]. 2005. Anuario estadístico del estado de Yucatan. Serie Anuarios estadísticos por entidad federativa. Gobierno del estado de Yucatán-INEGI. México.

Infante S. y Zárate-de-Lara G.P.. 2000. Métodos estadísticos, un enfoque interdisciplinario. Trillas. México.

Jiménez-Rojas E. M., Londoño-Vega A.C. y Vester H.F.M. 2002. Descripción de la arquitectura de Iryanthera tricornis, Osteophloeum platyspermum y Virola pavonis (Myristicaceae). Caldasia 24:65-94.

Kotz S., Read C.B., Balakrishnan N. y Vadakobic B. 2006. Encyclopedia of statistical science. Wiley-Interscience. New Jersey.

Küppers M. 1989. Ecological significance of above-ground architectural patterns in woody plants: a question of cost-benefit relationships. Trends in Ecology and Evolution 4:375-379.

Lauri P.E., Maguylo K. y Trottier C. 2006. Architecture and size relations: an essay on the Apple (Malus $\times$ domestica, Rosaceae)
Tree. American Journal of Botany 93:357-368.

Ledig F.T. 1983. The influence of genotype and environment on dry matter distribution in plants. En: Huxley P.A Ed. Plant Research and Agroforestry. Nairobi, Kenya, pp. 427-454 International Council for Research in Agroforestry. Kenya.

Levy-Tacher S. y Hernández-Xolocotzi E. 1994. Aprovechamiento forestal tradicional de los Hubches en Yucatán. En:HernándezXolocotzi E., Bello-Baltazar E. y Levy-Tacher S.I. Comp. La milpa en Yucatán, un sistema de producción agrícola tradicional Tomo I pp. 247-270. Colegio de Postgraduados, México.

Mäkelä A. y Valentine H.T. 2006. Crown ratio influence allometric scaling in trees. Ecology 87:2967-2972.

McMahon T. 1975. The mechanical design of trees. Scientific American 233:92-102.

Meza A. y Torres G. 2004. Efecto de la poda forestal en la calidad de la madera. Kurú Revista electrónica consultable en: <http:// www.tec.cr/sitios/Docencia/forestal/Revista_Kuru/anteriores/ anterior1/pdf/MEZA\%20Y\%20TORRES30feb04.pdf> Consultada por última vez septiembre 2009.

Moguel-Ordóñez Y., Echazarreta-Gonzalez C. y Mora-Escobedo R. 2005. Calidad fisicoquímica de la miel de abeja Apis mellifera producida en el estado de Yucatán durante diferentes etapas del proceso de producción y tipos de floración. Técnica Pecuaria de México 43:323-334.

Moreno N.P. 1984. Glosario botánico ilustrado. Instituto Nacional de Investigaciones sobre Recursos Bióticos, Compañía Editorial Continental. Xalapa, Veracruz. México.

Muñoz F., Espinoza M., Cansino J., Rubilar R. y Herrera M. 2008. Efecto de poda y raleo en el área foliar de Eucaliptus nitens. Bosque 29:44-51.

Normand F., Pambo-Bello A.K., Trottier C. y Lauri P.E.. 2009. Is axis position within tree architecture a determinant of axis morphology, branching, flowering and fruiting? An essay in mango. Annals of Botany 103:1325-1336.

Oldeman R.A.A. 1974. L'architecture de la forêt guyanaise. Mémoires ORSTOM 73. ORSTOM. Paris.

Osada N. y Takeda H. 2003. Branch architecture, light interception and crown development in saplings of a plagiotropically branching tropical tree, Polyalthia jenkinsii (Annonaceae). Annals of Botany 91:55-63.

Poorter L., Bongers L., Bongers F. 2006. Arquitecture of 54 moistforest tree species: traits, trade-off and functional groups. Ecology 87:1289-1301.

Ramírez-Cervantes M.A., González-Novelo S. A. y Sauri Duch E. 2000. Effect of the temporary thermic treatment of honey on variation of the quality of the same during storage. Apiacta 35:162-170.

SAS Institute Inc. 1999. User's guide, version 8. SAS Institute Inc. Cary, North Carolina.

Smith F.W. y Long J.N. 1989. The influence of canopy architecture on stemwood production and growth efficiency of Pinus contorta var. latifolia. Journal of Applied Ecology 26:681-691.

Sociedad Española de Ciencias Forestales [SECF]. 2005. Diccionario forestal. Mundi-Prensa. Madrid, España.

Sosa V., Gómez Pompa A. y Flores J.S. 1985. La flora de Yucatán. Ciencia y Desarrollo. 60:37-46.

Souza-Novelo N. 1981. Plantas meliferas y poliniferas que viven en Yucatan. Fondo Editorial de Yucatan. Mérida, Yucatán, México.

Standley P.C. y Steyermark J.A. 1946. Flora de Guatemala. Volu- 
men 24, parte IV. Chicago Natural History Museum. Chicago. Tomlinson P.B. 1978. Branching and axis differentiation in tropical trees. En: Tomlinson P.B. and Zimmermann M.H. Eds. Tropical trees as living systems pp: 187-207. Cambridge University Press. Cambridge, UK.

Tomlinson P.B. 1983. Tree architecture. American Scientist 71:141-149.

Tomlinson P.B. 1987. Architecture of tropical plants. Annual Review of Ecology and Systematics 18:1-21.

Tomlinson P.B. y Hill A.M. 1973. Growth habit of tropical trees: some guiding principles. En: Meggers B.J., Ayensu E.S. y Duckworth W.D. Eds. Tropical forest ecosystems in Africa and South America: a comparative review pp.129-143 Smithsonian Institute Press. Washington, DC.
Torquebiau E. 1981. Analyse architecturale de la forêt de Los Tuxtlas, Veracruz, Mexique. Tesis Doctoral. Université Montpellier II. Montpellier. $51 \mathrm{p}$.

Valdez-Hernández J.I. 2002. Aprovechamiento forestal de manglares en el estado de Nayarit, costa Pacífica de México. Madera y Bosques Número especial 2002:129-145.

Vester H.F.H. 2002. Modelos arquitectónicos en la flora arbórea de la Península de Yucatán. Boletín de la Sociedad Botánica de México 71:45-57.

White D.A. y Hood C.S. 2004. Vegetation patterns and environmental gradients in tropical dry forests of the northern Yucatan Peninsula. Journal of Vegetation Science 15:151-161.

Wilson B.F. 1984. The growing tree. The University of Massachusetts Press, Massachusetts.

Recibido: 17 de marzo de 2009

Aceptado: 1 de septiembre del 2009 


\section{Anexo A. Glosario.}

\section{1.- Clases de tamaño:}

Brinzales. Árboles jóvenes o plántulas que crecen vigorosamente y sin ramas secas, (SECF, 2005) menores a 1.5 m de altura (modificado de Valdez-Hernández, 2002).

Latizales. Árboles jóvenes que inician su poda natural (SECF, 2005), con una altura mayor o igual a $1.5 \mathrm{~m}$ y hasta $2.5 \mathrm{~cm}$ de diámetro a la altura del pecho (DAP = $1.3 \mathrm{~m}$ sobre el suelo) (modificado de Valdez-Hernández, 2002).

Fustales. Árboles maduros o adultos que se mantienen hasta el final de la vida de la masa forestal o bosque (SECF, 2005), tienen un DAP mayor de $2.5 \mathrm{~cm}$ (modificado de Valdez-Hernández, 2002).

2.- Crecimiento del eje principal (Tomlinson y Gill, 1973; Tomlinson, 1978, 1983; Hallé et al., 1978; Brayton, 1984; Barthélémy y Caraglio, 2007):

- Eje. Producto de un meristemo apical.

- Fuste. Tronco, formado de un eje principal (monopodio) o del encadenamiento de ejes (simpodio).

- Patrón:

Monopódico o excurrente, origina un tronco principal por la actividad de una yema apical.

Simpódico o delicuesente, tronco o rama formada por el encadenamiento de ejes con crecimiento determinado; se origina por la actividad de varias yemas axilares sucesivas. La yema apical inicial es sustituida por una yema axilar que produce una rama que crece verticalmente y el patrón continúa repitiéndose sucesivamente por acción de yemas axilares. Todas las ramas pueden alcanzar la misma altura.
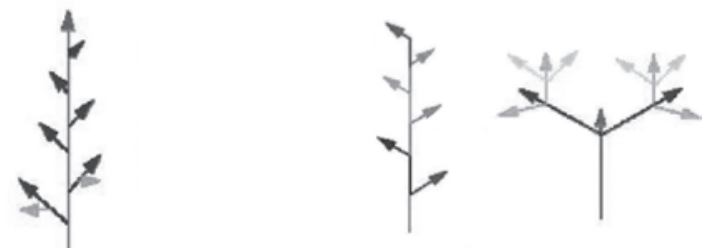

Imagen: http://www.scribd.com/doc/7241003/Exomorfolog-A

\section{- Forma:}

Determinado, el meristemo apical se convierte en una inflorescencia terminal, zarcillo o bola de parénquima (bayoneta), por lo que ahí se detiene el crecimiento del eje.

Indeterminado, sin fin determinado.

\section{- Actividad:}

Proléptico, surgido a partir de yemas latentes

Siléptico, originado de yemas sin latencia.

3.- Ramificación (Tomlinson y Gill, 1973; Tomlinson, 1978, 1983; Hallé et al., 1978; Brayton, 1984; Barthélémy y Caraglio, 2007):

\section{- Patrón:}

Monopodial. Origina un tallo principal por la actividad de una yema apical.

Simpodial, derivado de la actividad de una sola yema lateral en cada evento de crecimiento; en este caso se dan dos posibilidades: a) que las sucesivas yemas y ramas se formen alternadamente a cada lado del eje, con lo que se adquiere una conformación aproximadamente en zigzag sin un eje principal y, b) que las sucesivas yemas se formen siempre del mismo lado.

- Orientación y simetría (Tomlinson y Gill, 1973; Tomlinson, 1978, 1983; Hallé et al., 1978; Brayton, 1984; Barthélémy y Caraglio, 2007):

Ortotrópico, eje erecto, con orientación vertical, simetría radial, filotaxia helicoidal y que normalmente no forma flores. Plagiotrópico, los ejes son más o menos horizontales, con simetría dorsiventral, ramificados en dos direcciones y que frecuentemente florecen.

Combinado o mixto, un eje cambia la orientación presentando los dos tipos de ejes.

- Órdenes de ramificación con respecto al eje principal: Directamente del eje principal es orden 1 y en sucesión, segundo y tercero (McMahon, 1975).

- Ángulo de inserción de ramas (Moreno, 1984; Osada y Takeda, 2003):

Ascendente. Ángulo de 16 a $45^{\circ}$.

Difuso. Ángulo de 46 a $75^{\circ}$.

- Longitud de ramas y entrenudos (Osada y Takeda, 2003):

Largas. Que incrementan la longitud de las plantas.

Corta. Que tienden a sostener estructuras reproductoras. 


\section{Anexo A. Glosario. (continuación)}

4.- Estructuras reproductivas (Tomlinson y Gill, 1973; Tomlinson, 1978, 1983; Hallé et al., 1978; Brayton, 1984; Barthélémy y Caraglio, 2007):

- Posición:

Apical. En el extremo distal de la rama.

Axilar. En la axila de la rama y en cualquier posición por debajo del ápice.

- Inflorescencias:

Desarrollo: Acrópeto, que desarrolla desde la base hacia el ápice.

Morfología: Monopodiales Complejas: Panícula o panoja; es un caso especial de racimo doble, con ejes cerrados y con inflorescencias parciales complejas botrioides o monopodiales en la base, simplificadas hacia el ápice (disminuyen en número de flores y ramas).

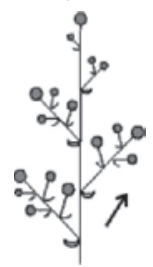

Simpodiales Complejas: Cimoides, desarrollan una o dos inflorescencias parciales en la axila de los profilos, inmediatamente por debajo de la flor terminal. Dichas inflorescencias pueden ser monocasios o dicasios. Simpodial monocasio: una sola inflorescencia parcial se desarrolla debajo de la flor terminal (imagen izquierda) y Simpodial dicasio: dos inflorescencias parciales se desarrollan debajo del eje principal (imagen derecha).
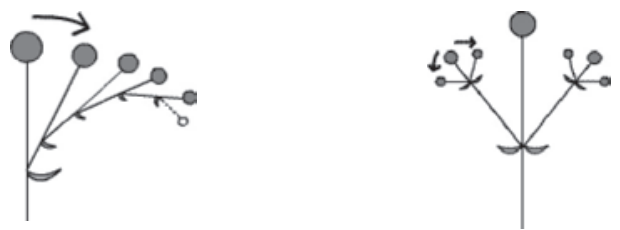

5.- Reiteración. Repetición de la forma de crecimiento dentro de la copa de un árbol, o repetición de la arquitectura primaria de un modelo de crecimiento dentro de un mismo individuo (Hallé et al., 1978; Tomlinson, 1978, 1987; Barthélémy y Caraglio, 2007).

Total. Repetición completa del modelo arquitectónico primario de crecimiento dentro de la copa de un individuo.

Parcial. Repetición parcial del modelo arquitectónico primario de crecimiento dentro de la copa de un individuo.

Adaptativa. Repetición del modelo arquitectónico primario de crecimiento dentro de la copa de un individuo, como respuesta a factores extremos.

Traumática. Repetición del modelo arquitectónico primario de crecimiento dentro de la copa de un individuo, como respuesta al daño provocado en el tronco principal o en la yema apical.

Proléptica. Surgida a partir de yemas latentes.

Siléptica. Originada de yemas sin latencia.

6.- Metamorfosis. Cambio de un modelo arquitectónico a otro, inducido por el cambio en la etapa de desarrollo del individuo (Hallé et al., 1978).

7.- DMS. Diferencia mínima significativa. Es el valor mínimo por el cual deben diferir dos medias para ser declaradas de poblaciones distintas (Infante y Zárate-de-Lara, 2000).

8.- Valores promedio. La media aritmética de $n$ observaciones de la variable $X$, se define como la suma de las observaciones entre el número total de estas (Infante y Zárate-de-Lara, 2000).

9.- Distancia de Mahalanobis. Parámetro que determina la distancia o separación entre dos poblaciones (Enciclopedia of Stadistical Science, 2006). 